\title{
A highly efficient numerical approach: extended cohesive damage model for predicting multicrack propagation
}

\author{
$\mathrm{X} \mathrm{Li}^{1}$ and $\mathrm{J} \mathrm{Chen}^{2}$,* \\ ${ }^{1}$ College of Shipbuilding Engineering, Harbin Engineering University, Harbin, China \\ ${ }^{2}$ School of Civil Engineering and Surveying, University of Portsmouth, Portsmouth, UK
}

\begin{abstract}
A highly efficient numerical approach: extended cohesive damage model (ECDM) for predicting multicrack propagation is introduced in this paper. The ECDM is developed within the framework of the eXtended Finite Element Method (XFEM). Unlike XFEM the enriched degrees of freedom are eliminated from the final condensed equilibrium equations in the ECDM. To account for the cohesive crack effect, an equivalent damage scalar relating to a strain field is introduced in terms of energy dissipation. The ECDM is capable of characterizing discontinuities with conventional degrees of freedom (DoFs) only, thus it is significantly efficient in modelling multicrack propagation. The basic formulations, numerical implementation and detailed investigation of the performance of the ECDM through modelling the selected benchmark specimens are given in this paper. This investigation shows the ECDM can effectively guarantee the convergent solutions in nonlinear fracture analysis and can efficiently reduce the computer CPU time in modelling selected fracture benchmark specimens by more than $60 \%$ compared to the XFEM in ABAQUS. Therefore, the ECDM is a robust computational approach for predicting multicrack failure mechanism in engineering materials and structures.
\end{abstract}

Keywords: ECDM, XFEM, computing efficiency, damage, multicrack propagation

${ }^{*}$ Corresponding author.

E-mail address: jiye.chen@port.ac.uk (J. Chen). 


\section{Introduction}

The Numerical simulation of discontinuities and its evolution via finite element method (FEM) is currently one of the major concerns in computational fracture mechanics. In most FEM based approaches, there are two classical technologies in modelling discontinuities: the cohesive zone model (CZM) [1-4], and the adaptive mesh refining (AMR) approach [5-7]. In CZM based modelling, discontinuities must be limited at inter-element boundaries. Thus in the discretization stage, discontinuities must align with element boundaries. Obviously, the a priori knowledge of discontinuity path is necessary for numerical characterization. Recently a breakable CZM model was developed which is declared to model arbitrary crack propagation, but the crack path is still dependent on the mesh structure [8]. By real-timely modifying mesh topology, the AMR approach can adaptively and explicitly model a discontinuity. Nevertheless, to capture a propagating discontinuity, the self-adaption of a mesh in the AMR approach is computationally expensive in non-linear fracture analysis [6]. Therefore, the above two computational modelling methods of propagating discontinuities have long been restricted by the mesh bias dependences and significant computational burden. These deficiencies hinder their further applications in engineering fields.

In the past decade, based on the Partition of Unity Method (PUM), a rapid development which can address the aforementioned drawbacks has been made to conduct the arbitrary discontinuity problems within continuum solids [10-14]. Among all these PUM based approaches, the eXtended Finite Element Method (XFEM), as a combination of the classical FEM and PUM, was originally introduced by Belytschko et al. [10] and subsequently enhanced by Moës et al. [15]. By enriching the classical piecewise polynomial approximation basis within a FEM framework, XFEM is capable of thoroughly conducting computational characterization for non-smooth features within a discontinuous medium. The existence of discontinuity can be freely laid within elements, irrespective of its size and specific orientation. Thus, no mesh regeneration is needed during discontinuity evolution, and the element boundary would no longer need to be the discontinuity surface, which is a significant benefit to the work of modelling crack propagation. Then, typical drawbacks of traditional finite elements in 
modelling displacement discontinuities, e.g. spurious mesh size and mesh bias dependences, can be effectively overcome. XFEM has been applied to model cohesive cracks of mechanical metamaterials [16, 17], fatigue damage problems [18, 19], fluid mechanics problems [20, 21], thermal problems [22] and delamination [23] and matrix fracture [24, 25] in composites, etc.

Besides, some other discrete discontinuity approaches based on PUM were developed as well. Hansbo et al. presented a phantom-node method to model arbitrary discontinuities. In their approach, the external nodes named 'phantom nodes' are placed for the description of locally non-polynomial phenomena. According to Areias and Belytschko [26], the phantom node method is just another implementation of XFEM. Working within the variation framework, K. Garikipati carried out the Variation Multiscalar Cohesive Method (VMCM) [27-29] to model the crack propagation in isotropic solid and laminated composites. The key idea of VMCM is the identification of a fine scalar field with a component of the displacement that has a large gradient. Oliver et al. [30] investigated an embedded finite element method (E-FEM) which is an alternative approach for XFEM. A comparative study [31] was carried out between E-FEM and XFEM, which demonstrated that not only the numerical accuracy but also the efficiency of E-FEM is evidently improved by the implementation of elemental enrichment rather than nodal enrichments required by XFEM. Wu et al. [32] enhanced E-FEM by combining the advantages of XFEM and E-FEM. On one hand, a non-uniform discontinuity mode is considered as in XFEM. On the other hand, the condition of traction continuity is accounted in the statically optimal form as in most E-FEMs. All these discrete discontinuity models are essentially formulated with enriched continuous displacement modes in previous finite elements by given additional discontinuous displacements for capturing the physical discontinuity i.e. fractures, cracks, slip lines, etc.

The major drawback of the above mentioned PUM related methods is the enriched terms because additional DoFs or nodes certainly bring expensive computational cost. Sometimes, it tends to be even impossible to achieve convergent solution when encountering highly strong 
nonlinearity [33]. In consideration of this drawback, a novel Extended Cohesive Damage Model (ECDM) was recently developed by the authors to describe the arbitrary crack propagation in engineering materials [34]. The purpose of this work is to introduce the numerical implementation and assess the performance of the ECDM in characterizing the arbitrary crack propagation in engineering materials. The superiority of the ECDM in computational accuracy, efficiency and robustness is evaluated in detail by modelling selected fracture benchmark tests. The computational efficiency of the developed ECDM will be demonstrated through the comparison with XFEM predictions. Through these modelling predictions of selected fracture benchmark tests, the high efficiency of the ECDM is proved.

\section{Theoretical fundamentals of the ECDM}

\subsection{Kinematics of cohesive crack problem}

In a $2 \mathrm{D}$ continuum $\mathfrak{R}^{2}$, consider a discontinuous physical domain $\Omega \in \mathfrak{R}^{2}$, whose outward normal vector $\mathbf{n}$, intersected by a cohesive crack $\Gamma_{\mathrm{d}}$ with normal vector $\mathbf{m}$, as shown in Fig. 1(a). Such that the domain is divided into two subdomains represented as $\Omega^{+}$and $\Omega^{-}$, respectively, as shown in Fig. 1(b). The crack results in the presence of two new consistent internal boundaries $\Gamma_{\mathrm{d}}^{+}$and $\Gamma_{\mathrm{d}}^{-}$. The prescribed external load $\overline{\mathbf{t}}$ is imposed on boundary $\Gamma_{\mathrm{t}}$ and the displacement $\overline{\mathbf{u}}$ is assumed on boundary $\Gamma_{\mathrm{u}}$. Omitting the body forces, the strong form of the equilibrium equation can be written as:

$$
\operatorname{Div} \sigma=0
$$

where the second-order tensor $\boldsymbol{\sigma}$ denotes the stress field in the bulk domain $\Omega=\Omega^{+} \cup \Omega^{-}$. The boundary conditions for the domain $\Omega$ are:

$$
\begin{aligned}
\boldsymbol{\sigma} \cdot \mathbf{n} & =\overline{\mathbf{t}}\left(\text { on } \Gamma_{\mathrm{t}}\right) \\
\mathbf{u} & =\overline{\mathbf{u}}\left(\text { on } \Gamma_{\mathrm{u}}\right)
\end{aligned}
$$

Due to the presence of cohesive segment, boundary conditions imposed on the discontinuous boundaries $\Gamma_{\mathrm{d}}^{+}$and $\Gamma_{\mathrm{d}}^{-}$are given below.

$$
\begin{gathered}
\mathbf{t}^{+}=\boldsymbol{\sigma}^{+} \cdot \mathbf{n}^{+}=-\mathbf{t} \quad\left(\text { on } \Gamma_{\mathrm{d}}^{+}\right) \\
\mathbf{t}^{-}=\boldsymbol{\sigma}^{-} \cdot \mathbf{n}^{-}=\mathbf{t} \quad\left(\text { on } \Gamma_{\mathrm{d}}\right)
\end{gathered}
$$


According to the traction-separation law [1], the traction attributing to the cohesive segment between crack surfaces can be obtained from the relative displacement:

$$
\mathbf{t}=\mathbf{t}(\delta)
$$

in which $\delta$ is the relative displacement between two boundaries of discontinuity. The above equation serves as a nonlinear material model when discontinuity onsets.

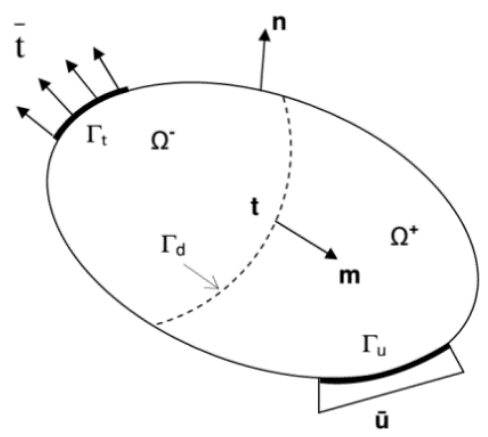

a

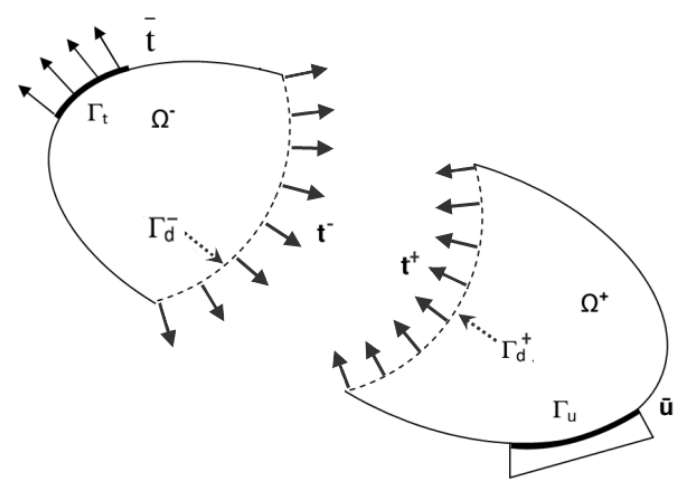

b

Fig 1 . Notation for a 2D domain with an arbitrary discontinuity $\Gamma_{\mathrm{d}}$.

While the cohesive tractions are present within the specified segment of crack, the total potential of the body should take account for the contribution from cohesive tractions transferred through the crack surface. According to the principle of virtual work, the weak form of equilibrium equation based on the strong form of the equilibrium equation given in Eq. (1) can be written as:

$$
\int_{\Omega}[\nabla \boldsymbol{\omega}(\mathbf{x}): \sigma(\mathbf{u}(\mathbf{x}))] d \Omega-\int_{\Gamma_{t}} \boldsymbol{\omega}(\mathbf{x}) \cdot \overline{\mathbf{t}} d \Gamma-\int_{\Gamma_{d}^{+}} \boldsymbol{\omega}(\mathbf{x}) \cdot \mathbf{t}^{+} d \Gamma-\int_{\Gamma_{d}^{-}} \boldsymbol{\omega}(\mathbf{x}) \cdot \mathbf{t}^{-} d \Gamma=0
$$

In the weak form, $\boldsymbol{\omega}(\mathbf{x})$ and $\mathbf{u}(\mathbf{x})$ are test and solution functions, respectively.

\subsection{Displacement field and shifted Heaviside function}

Chen [35] has recently investigated the basic concept of combing XFEM with CZM without using a specified enriched item to cope with the singularity problem at the crack-tip. The XFEM test function of the discontinuous displacement field can be given as [16]: 


$$
\mathbf{u}\left(\mathbf{x} \neq \mathbf{u}^{\mathrm{s}}(\mathbf{x})+S t e p_{\mathrm{d}}(\mathbf{x}) \mathbf{a}(\right.
$$

where $\mathbf{u}^{\mathrm{s}}(\mathbf{x})$ and $\mathbf{a}(\mathbf{x})$ stand for the regular displacement field and the displacement jump, respectively. $\operatorname{Step}_{\Gamma_{d}}(\mathbf{x})$ is a step function characterizing the physical jump when the element is completely separated (strong discontinuities). In FEM discrete form, the approximation of displacement field can be represented as:

$$
\mathbf{u}^{h}(\mathbf{x})=\sum_{i \in I} N_{i}(\mathbf{x}) \mathbf{u}_{\mathbf{i}}+\sum_{i \in I} N_{j}(\mathbf{x})\left(\mathscr{H}_{\Gamma_{d}}(\mathbf{x})-\mathscr{H}_{\Gamma_{d}}\left(\mathbf{x}_{\mathbf{i}}\right)\right) \mathbf{a}_{\mathbf{i}}
$$

in which $\mathscr{H}_{\Gamma_{d}}(\mathbf{x})=\left\{\begin{array}{ll}1, & \forall \mathbf{x} \in \Omega^{+} \\ 0, & \forall \mathbf{x} \in \Omega^{-}\end{array}\right.$is a Heaviside step function. It should be noted that, in the above discretization of displacement, the shifted function $\mathscr{H}_{\Gamma_{d}}(\mathbf{x})-\mathscr{H}_{\Gamma_{d}}\left(\mathbf{x}_{\mathbf{i}}\right)$ is introduced for satisfying the Kronecker- $\delta$ property (i.e., $N_{i}\left(x_{j}\right)=\delta_{i j}$ ). The introduction of a shifted Heaviside function does not alter the approximating basis while simplifying the implementation attributing to that resulting enrichment vanished in elements which are not cut by the discontinuity [19].

\subsection{Discrete and condensed equilibrium equations}

Using the weak form of the equilibrium equation from the Bubnov-Galerkin method, the discrete form of the equilibrium equation for static analysis can be written as shown in Eq. (10).

$$
\left[\begin{array}{ll}
\mathbf{K}^{u u} & \mathbf{K}^{u a} \\
\mathbf{K}^{a u} & \mathbf{K}^{a a}
\end{array}\right]\left[\begin{array}{l}
\mathbf{u} \\
\mathbf{a}
\end{array}\right]=\left[\begin{array}{l}
\mathbf{f}_{\text {ext }}^{u} \\
\mathbf{f}_{\text {ext }}^{a}
\end{array}\right]
$$

where $\mathbf{K}^{u u}$ and $\mathbf{K}^{a a}$ are the stiffness matrices associated with the standard FE approximation and the enriched approximation, respectively; $\mathbf{K}^{u a}$ or $\mathbf{K}^{a u}$ account for the coupling between the standard FE approximation and the enriched approximation; $\mathbf{f}_{e x t}^{\mathbf{u}}$ and $\mathbf{f}_{\text {ext }}^{\mathbf{a}}$ are the equivalent nodal force vectors for standard FEM DoFs and enriched DoFs, respectively; u denotes the standard DoFs while a denotes the enriched DoFs.

As aforementioned, the crack shown in Fig. 1 is a cohesive crack, and the discontinuous boundary is a cohesive crack boundary. Thus, in Eq. (10), the equivalent nodal force vectors can be expressed as: 


$$
\begin{aligned}
\mathbf{f}_{e x t}^{u} & =\int_{\Gamma^{\mathrm{h}}} \mathbf{N}^{T} \overline{\mathbf{t}} \mathrm{d} \Gamma \\
\mathbf{f}_{e x t}^{a} & =\int_{\Gamma^{\mathrm{h}}} \mathbf{N}^{T}\left(\mathscr{H}_{\Gamma_{d}}(x, y)-\mathscr{H}_{\Gamma_{d}}(x, y)\right) \overline{\mathrm{t}} \mathrm{d} \Gamma+\mathbf{f}_{c o h}
\end{aligned}
$$

Because of the existence of cohesive segment, the internal nodal force vector due to cohesive traction $\mathbf{t}$ on the crack surface $\Gamma_{d}$ can be expressed as:

$$
\begin{aligned}
\mathbf{f}_{c o h} & =\int_{\Gamma_{\mathrm{d}^{+}}}\left(\mathscr{H}_{\Gamma_{d}}(x, y)-\mathscr{H}_{\Gamma_{d}}\left(x_{i}, y_{i}\right)\right) \mathbf{N}^{T} \mathbf{t} d \Gamma+\int_{\Gamma_{\mathrm{d}^{-}}}\left(\mathscr{H}_{\Gamma_{d}}(x, y)-\mathscr{H}_{\Gamma_{d}}\left(x_{i}, y_{i}\right)\right) \mathbf{N}^{T}(-\mathbf{t}) d \Gamma \\
& =\int_{\Gamma_{\mathrm{d}}} \mathbf{N}^{T} \mathbf{t} d \Gamma
\end{aligned}
$$

The integration of the internal nodal force vector due to cohesive traction $\mathbf{t}$ shown in Eq. (12) can be obtained by numerical integration regarding to the cohesive stress on the crack surface $\Gamma_{d}$. In this work, the standard Gaussian integration scheme is used, that is,

$$
\mathbf{f}_{c o h}=\int_{\Gamma_{\mathrm{d}}} \mathbf{N}^{T} \mathbf{t} d \Gamma=\sum_{\mathrm{i}=1}^{\mathrm{n}} \mathbf{N}^{T}\left(\xi_{i}\right) \mathbf{t}\left(\xi_{i}\right) \mathrm{w}\left(\xi_{i}\right)
$$

where $\xi_{\mathrm{i}}$ is the coordinate of the Gauss integration point $i(1 \leq i \leq n) ; \quad \mathrm{w}\left(\xi_{i}\right)$ is a weight function. Substituting the expression for the equivalent nodal force vector in Eq. (11) into Eq. (10) results in Eq. (14):

$$
\left[\begin{array}{ll}
\mathbf{K}^{u u} & \mathbf{K}^{u a} \\
\mathbf{K}^{a u} & \mathbf{K}^{a a}
\end{array}\right]\left[\begin{array}{l}
\mathbf{u} \\
\mathbf{a}
\end{array}\right]=\left[\int_{\Gamma^{\mathrm{h}}} \mathbf{N}^{T}\left(\mathscr{A}_{\Gamma_{d}}(x, y)-\mathcal{A}_{\Gamma_{d}}\left(x_{i}, y_{i}\right)\right) \overline{\mathrm{t}} \mathrm{d} \Gamma\right]+\left[\begin{array}{c}
0 \\
\mathbf{f}_{c o h}
\end{array}\right]
$$

To be able to reach a fully condensed equilibrium system, the additional enrichment term $\mathbf{a}$ is eliminated, thus the equilibrium equation with the standard FEM unknown quantities can be consequently obtained as shown in Eq. (15):

$$
\left(\mathbf{K}^{u u}-\mathbf{K}^{u a}\left(\mathbf{K}^{a a}\right)^{-1} \mathbf{K}^{a u}\right) \mathbf{u}=\mathbf{f}_{e x t}^{u}-\mathbf{K}^{u a}\left(\mathbf{K}^{a a}\right)^{-1} \mathbf{M} \mathbf{f}_{e x t}^{u}-\mathbf{K}^{u a}\left(\mathbf{K}^{a a}\right)^{-1} \mathbf{f}_{c o h}
$$

where, $\mathbf{M}$ is a transformation matrix to link the $\mathbf{f}_{e x t}^{a}$ and $\mathbf{f}_{e x t}^{u}$ as below.

$$
\mathbf{f}_{e x t}^{a}=\mathbf{M f}_{e x t}^{u}
$$


The exact expression for M can be found in the authors' previous work [34]. It should be noted that in most cases $\mathbf{M}$ is zero because there is no external load applied on the cohesive crack. Thus Eq. (15) can be rewritten as:

$$
\left(\mathbf{K}^{u u}-\mathbf{K}^{u a}\left(\mathbf{K}^{a a}\right)^{-1} \mathbf{K}^{a u}\right) \mathbf{u}=\mathbf{f}_{e x t}^{u}-\mathbf{K}^{u a}\left(\mathbf{K}^{a a}\right)^{-1} \mathbf{f}_{c o h}
$$

This ECDM formulation is a lower order equilibrium system compared to the XFEM in ABAQUS, which permits nodal displacement calculation of the cracked element using standard FEM DoFs only. This proposed rigorous mathematical procedure can fully cover the damage evolution from a weak discontinuity to a strong discontinuity.

\subsection{Cohesive damage model for the discontinuity}

The cohesive damage law is employed here to characterize the nonlinear cohesive segment evolution transferred across the discontinuity. The cohesive traction $\mathbf{t}_{c o h}=\left[t_{n}, t_{s}\right]^{T}$ contains two components, which are normal traction and shearing traction, respectively. As a function of the damage scalar $d$ in both normal and tangential directions at crack surface, $\mathbf{t}_{c o h}$ decreases monotonically to zero, which is mathematically expressed by Eq. $(18)[1,35]$.

$$
\mathbf{t}_{c o h}=(1-d) \mathbf{t}_{0}
$$

where $\mathbf{t}_{0}$ is the traction when the crack initiates. The cohesive tractions in Eq. (18) are calculated at the crack surface in the directions $n$ and $s$, respectively. In the implementation, the transformation of coordinates from local coordinate $n$ and $s$ to global coordinate $x$ and $y$ is necessarily required and expressed by Eq. (19).

$$
\mathbf{t}_{\text {coh }}^{\text {global }}=[\mathbf{R}] \mathbf{t}_{c o h}^{\text {local }}
$$

where the transformation matrix $\mathbf{R}$ is given by Eq. (20).

$$
[\mathbf{R}]=\left[\begin{array}{cc}
-\sin \theta & \cos \theta \\
\cos \theta & \sin \theta
\end{array}\right]
$$

where $\theta$ is the angle between the coordinate $n$-s and the coordinate $x-y$. There is not a physical relative displacement $\Delta \delta$ before a crack forms within the elements. Two schemes are widely used in approximating the evolution of cohesive failure, which are the linear softening scheme 
and the exponential softening scheme, respectively, as shown with the traction-relative displacement $\Delta \delta$ functions in Fig. 2. Nevertheless, as demonstrated in the ECDM equilibrium, eliminating the enriched DoFs has resulted in a vanishing relative displacement $\Delta \delta$ in the ultimate solution, which brings difficulties in recognizing the softening status of cohesion.
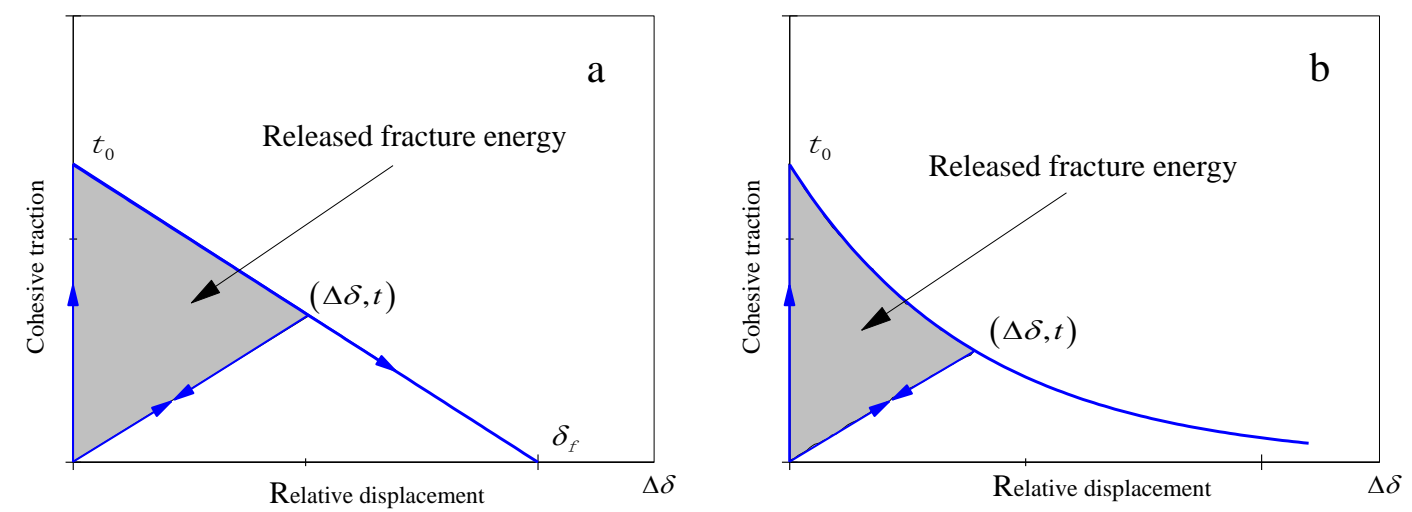

Fig. 2. Cohesive damage law: (a) linear softening and (b) exponential softening.
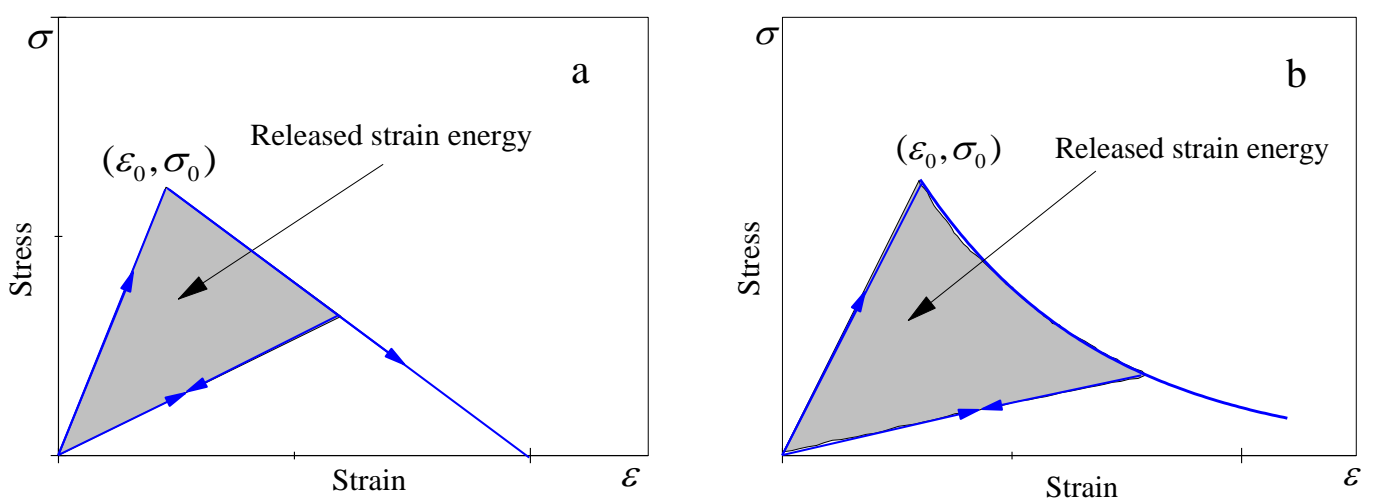

Fig. 3. Material constitutive behavior: (a) linear softening scheme; (b) exponential softening scheme.

In this developed ECDM, a new equivalent damage scalar based on strain energy dissipation is used to avoid the appearance of the additional DoFs related displacement gap $\Delta \delta$. In the ECDM based FEM modelling, it is expected that evolution of the cohesive zone will present a micro behavior of strain softening, so that the strain energy dissipation shown in Fig. 3 due to the occurrence of fracture is equivalent to the released work done by cohesive traction or released fracture energy shown in Fig. 2. Based on this consideration, the macro performance of material 
with the bilinear cohesive softening law and the exponential cohesive softening law follow the specified schemes with similar curve shapes, as demonstrated in Fig. 3a and Fig. 3b, respectively. Therefore, in the utilization of the ECDM, the damage scalar can be expressed equivalently by a released strain energy using the linear softening or the exponential softening law as shown in Eq. (21).

$$
\begin{aligned}
& d=\frac{\int_{\Omega} \frac{1}{2} \sigma^{0} \varepsilon d \Omega-\int_{\Omega} \frac{1}{2} \sigma \varepsilon^{0} d \Omega}{G_{c} l_{\text {crack }}} \\
& \text { or } \\
& d=\frac{\int_{\Omega} A\left\{\exp \left[\frac{\sigma^{0}}{A}\left(\varepsilon^{0}-\varepsilon\right)\right]-1\right\} d \Omega+\int_{\Omega} \frac{1}{2}\left(\sigma^{0} \varepsilon^{0}-\sigma \varepsilon\right) d \Omega}{G_{c \text { cra } l_{k}}}
\end{aligned}
$$

where, $\sigma^{0}$ and $\varepsilon^{0}$ are the material strength and the initial damage strain respectively at the onset of damage accumulation; $l_{\text {crack }}$ is elemental crack length from the start point to the end point of the crack in a cracked element. The damage status is considered to be coincident along the elemental crack length; $G_{c}$ is fracture energy; $A$ is a parameter expressed as $\frac{G_{c} l_{\text {crack }}}{S}-\frac{1}{2} \sigma^{0} \varepsilon^{0}$ in which $S$ is the total area under the curve of the softening damage law shown in Fig. 3. Herein a softening constitutive law is used for reducing the cohesive traction, i.e. $\sigma=(1-d) \sigma^{0}$. Bringing this calculation into Eq. (21), an explicit expression of the equivalent damage scalar can be achieved as shown below:

$$
\begin{aligned}
& d=\frac{\int_{\Omega} \frac{1}{2} \sigma^{0} \varepsilon d \Omega-\int_{\Omega} \frac{1}{2} \sigma^{0} \varepsilon^{0} d \Omega}{G_{c} l_{\text {crack }}-\int_{\Omega} \frac{1}{2} \sigma^{0} \varepsilon^{0} d \Omega} \\
& \text { or } \\
& d=\frac{\int_{\Omega} A\left\{\exp \left[\frac{\sigma^{0}}{A}\left(\varepsilon^{0}-\varepsilon\right)\right]-1\right\} d \Omega+\int_{\Omega} \frac{1}{2} \sigma^{0}\left(\varepsilon^{0}-\varepsilon\right) d \Omega}{G_{c} l_{\text {crack }}-\int_{\Omega} \frac{1}{2} \sigma^{0} \varepsilon d \Omega}
\end{aligned}
$$

It should be noted that the damage scalar $d$ in Eq. (22) is a function of the strain field obtained with conventional DoFs only, with which the requirement to calculate the enriched DoFs related displacement gap $\Delta \delta$ can be effectively avoided. 


\section{Numerical implementation}

In most existing discrete discontinuity approaches, a vital requirement for efficient implementation is the FEM code which allows flexible variation within limited number of DoFs per node and number of integration points per element. Attributing to the elimination of enriched DoFs, the ECDM can overcome this restriction and be easily integrated into commercial FEM software. Another advantage of the ECDM is that it can relieve users from utilizing a sophisticated global tracking algorithm, such as the level-set functions in XFEM for a propagating discontinuity. Consequently, large pre-calculations can be avoided when a discontinuity is activated in a narrow band manner e.g. crack propagation in a local region. Nevertheless, it is still necessary to track the crack path so that the inter-element continuity of a discontinuity can be enforced. This can be carried out by a common block which is accessible to all user elements, within which the geometric information of a discontinuity can be constantly updated as it propagates. Herein, a local tracking algorithm based on element connections/graph is adopted in numerical implementation. When an element meets the criterion, the discontinuity propagates within that element along a straight line with the determined orientation, from the discontinuity starting intersection to the end one. Fig. 4 presents the cracked element topology for different approaches including XFEM, the ECDM and CZM. It can be seen from Fig. 4 that the ECDM (Fig. 4b) is only with standard DoFs u, while for XFEM (Fig. 4a), an additional DoFs a is contained at each node. In the case of CZM shown in Fig. 4c, although all the nodes are with the standard DoFs, the embedding cohesive element requires the introduction of new nodes, which essentially increases the number of DoFs in a FE model. The approximations of the element deformation on the presence of a crack using different schemes are also illustrated in Fig. 4. In the ECDM scheme, the nodal displacements can be solved accurately, but the physical displacement gap (the dash lines in Fig. 4b) cannot be presented by nodal displacements. Instead, it can be presented by a strain field with a distinguished value. 

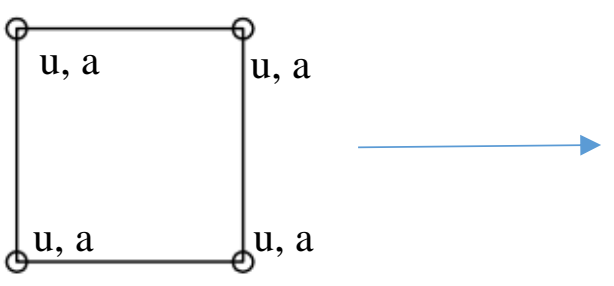

(a)

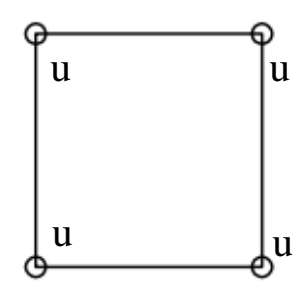

(b)

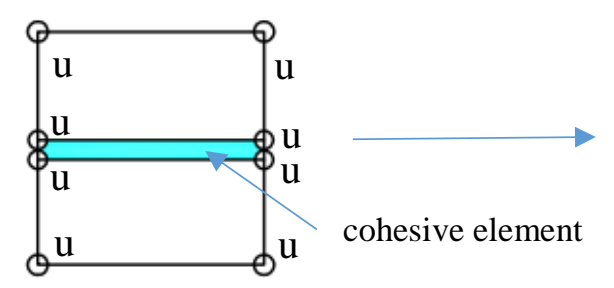

(c)
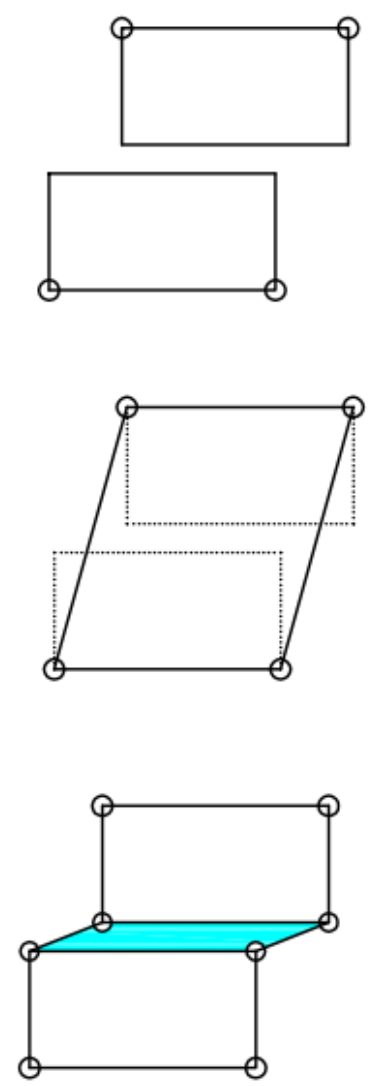

Fig. 4. The characterization of a crack by different approaches: (a) XFEM, (b) The ECDM and (c) CZM.

Identifying the element in which a discontinuity propagates and establishing the discontinuity configuration are required at the beginning of tracking discontinuity propagation. Determination of discontinuity nucleation is the first work. Nucleation is allowed to happen within any potential elements in the computationally accounted domain, whereas the propagation is originated at the front element of an existing discontinuity tip. In the numerical implementation, a discontinuity nucleation in an element is accounted at its midpoint where the maximum principal stress is over the failure criteria. The perpendicular direction to the direction of the maximum principal stress is determined as the crack direction. The determined crack starts from the middle point and ends at the edge of the cracked element through a straight line along the crack direction. In the case of existing cracks, normally, the existing crack should propagate to the element at the front of the crack tip. In the case of the cracked element at the boundary of the computational domain, only one tip point will be presented. Fig. 5 shows the 
crack propagation scheme in the ECDM implementation in which the red line is an existing crack. Considering the situation in Fig. 5(a), if the stress state of the element at the front crack tip satisfies the failure criterion, then the existing crack propagates across that element. Thus the previous crack tip is recognized as a start point of the crack in the new cracked element. A restriction given by a kinking angle between $45^{\circ}$ and $-45^{\circ}$ degree measured from the existing crack direction provides a potential propagation area as shown in the blue area in Fig. 5. In fact, this kinking restriction is a usual numerical treatment to avoid a possible crack curve back, for example, the XFEM model in ABAQUS also sets \pm 45 degree as a kinking restriction. Assuming a crack propagates along a straight line within the failed element, once the coordinates of the start point of the new crack is known, the end point of the crack can be trivially determined in terms of the continuity of the cracking path and the propagating orientation. This can be seen in Fig. 5(b). When crack propagation in an element is approximated, the discontinuity information will be updated for evaluating other elements.
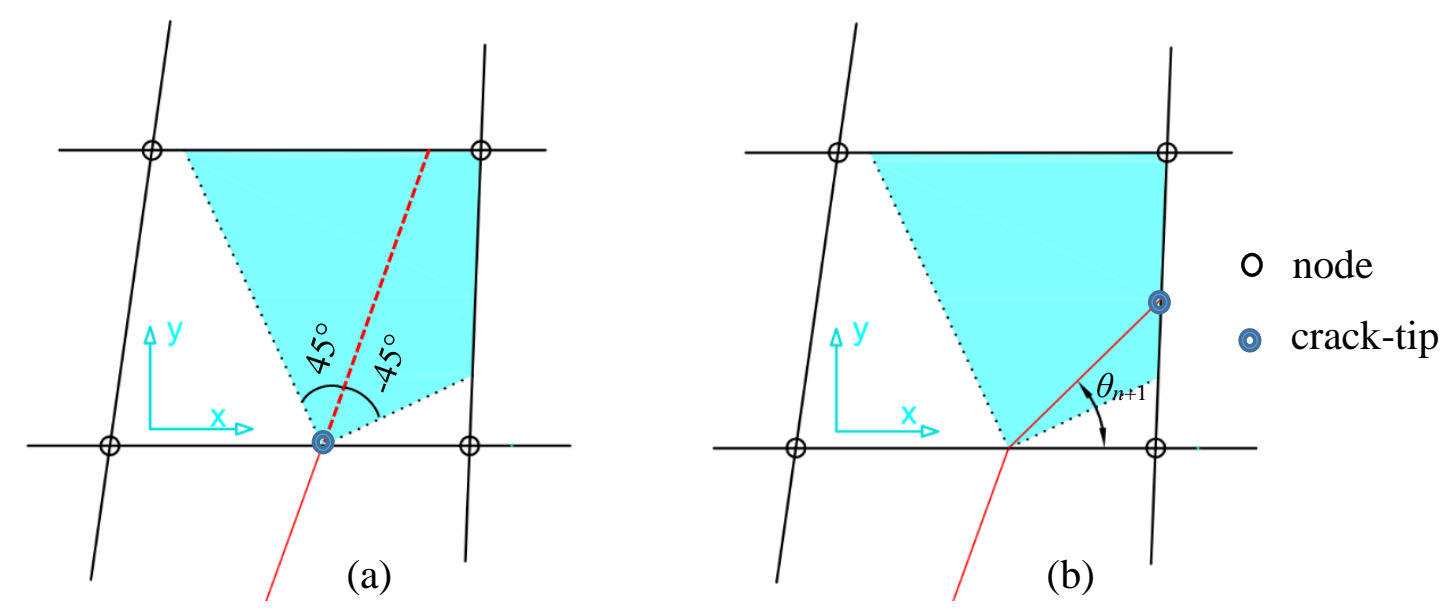

Fig. 5. The crack propagation scheme in the ECDM implementation. 


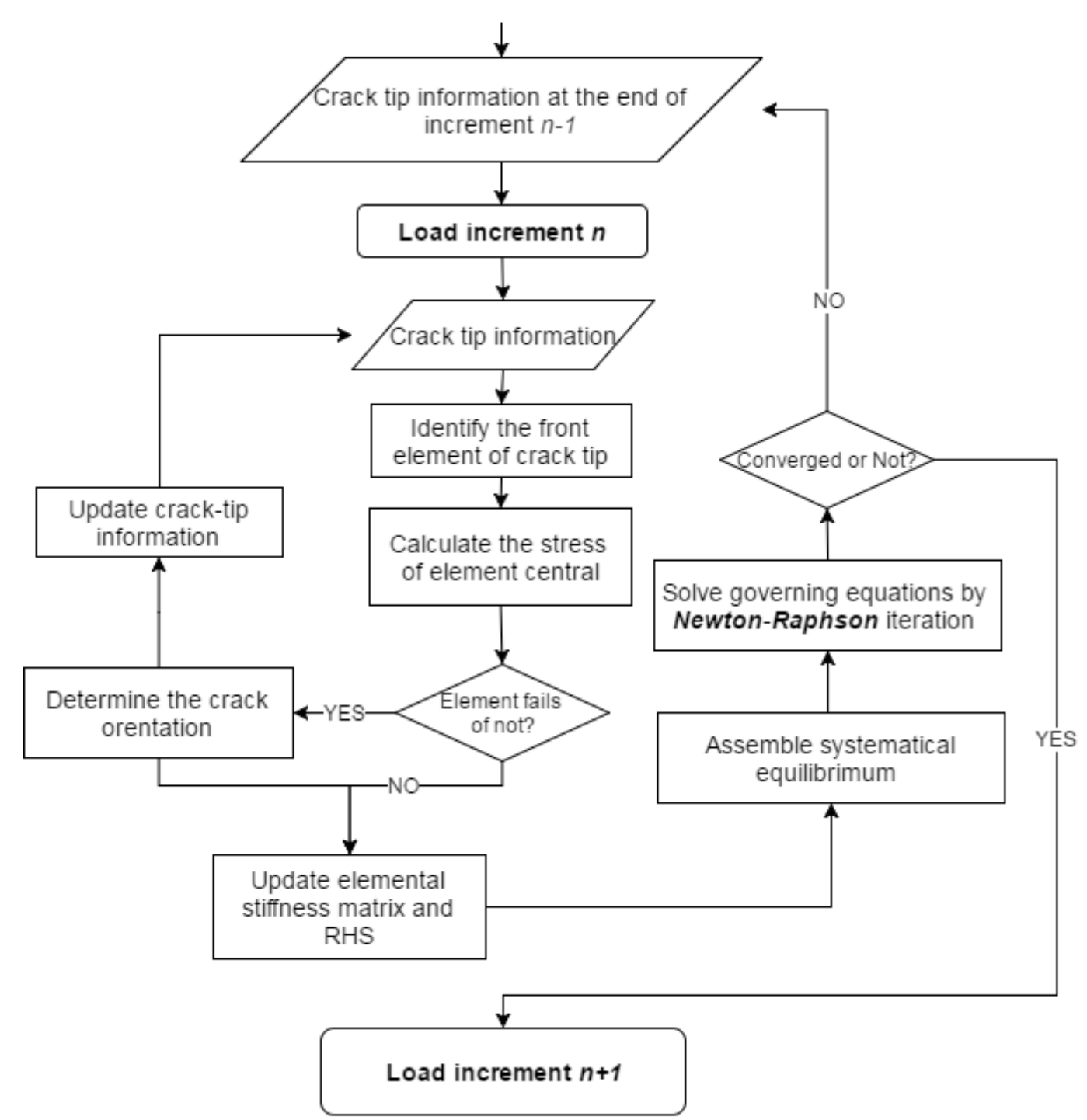

Fig. 6. The flow chart of crack propagation scheme and solving procedure in ECDM.

In the implementation of the most discrete discontinue approaches such as XFEM, in order to avoid introducing new DoFs into non-equilibrium states (e.g., in an iterative procedure) and to preserve the quadratic convergence rate of the Newton-Raphson scheme, the propagation is accounted after the iterative procedure performed [32]. Consequently, the increment size must be sufficiently small during the loading process, especially around the loading point of material failure, so as to capture the peak value and to avoid overestimation of element strength. In this work, attributing to the elimination of enriched DoFs, discontinuities are allowed to be initiated before iteration in the increment rather than at the end of a converged loading increment, which 
identifies with the normal nonlinear solving procedure and obtains accurate results even if a relatively coarse increment size is used.

The discontinuity propagation procedure according to the theoretical formulations of the ECDM presented in Section 2 has been implemented into FEM package ABAQUS by a user element UEL. Fig. 6 shows the flow chart of the UEL which uses the information for existing cracks, trail incremental displacements applied to the structure at the start of increment $n$, and the trail stress $\boldsymbol{\sigma}$ at the front of crack-tip of the identified element for evaluating crack propagation. If a crack is judged to propagate, the elemental crack configuration will be updated with computed new crack orientation and geometric information of previous crack tip. The updated crack information includes the location of the crack within the individual element and, in particular, the nodes for the starting and end points of the crack located at elemental edges. Subsequently, the element stiffness matrix and the nodal residual force can be updated according to Eq. (15) or Eq. (17). In the general route to solve nonlinear equilibrium equations, the Newton-Raphson iteration method associated with a line search algorithm is performed. When the residual nodal force reduces to the prescribed tolerance, the convergent solution is obtained. Subsequently, the computing process moves to increment $n+l$ with newly updated crack information. In the case of convergence failure, the computing system will automatically adjust the size of increment $n$, and repeat the above procedure using the crack information backed up at the end of increment $n-1$, until reaching the convergent solution. Since the crack is tracked in real time and each element can access crack information at any time, the crack can propagate through more than one element within one increment.

\section{Numerical applications}

\subsection{Single-edge notched beam}

A comparative study is carried out on a benchmark test using both ECDM and XFEM to assess the performance of the ECDM approach regarding its accuracy and efficiency in the prediction of multicrack propagation. The XFEM model in the FEM commercial package ABAQUS is 
adopted in this comparative study. In the test work [36], a single notched beam as shown in Fig. 7 is subjected to four-point bending. A mixed failure mode with a curved cracking path was observed experimentally. The dimensions of the beam is $440 \times 100 \times 100 \mathrm{~mm}^{3}$; a notch with the size $5 \times 20 \times 100 \mathrm{~mm}^{3}$ is located at the top center. The loading and boundary conditions can be seen from Fig. 7. A rigid bar between two loading points is set up for the purpose of imposing a proportionally distributed load $P$ at the bottom of beam. Through the rigid bar, the proportional loads applied on the left and right loading point are $P / 11$ and $10 P / 11$, respectively. The analysis can be material independent, following the previous test work given by references [16] and [32], the material properties used in modelling are: Young's modulus $E=35000 \mathrm{MPa}$, Poisson's ratio $v=0.2$, tensile strength $f_{t}=3.0 \mathrm{MPa}$ and fracture energy $G c=0.1 \mathrm{~N} / \mathrm{mm}$.

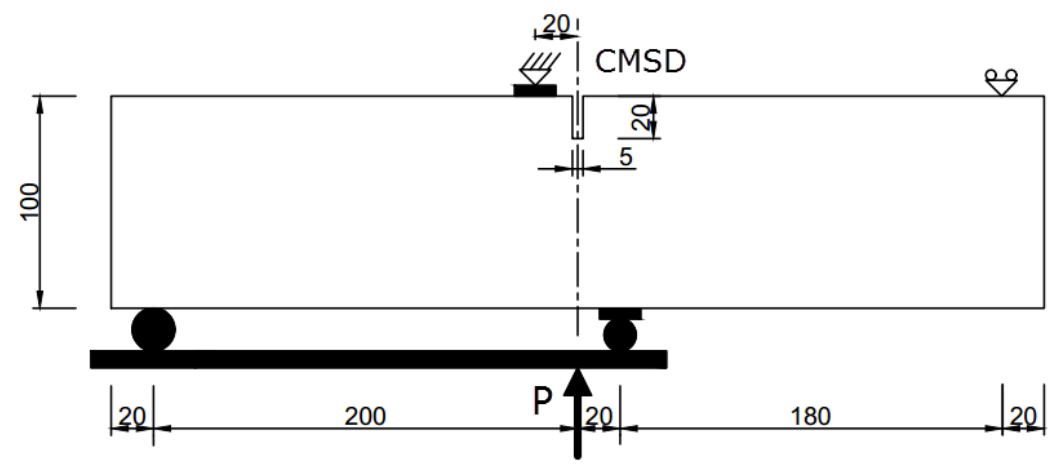

Fig. 7. Configuration of the Single-notched beam under four points bending.

Four meshes, named S1, S2, S3 and S4 with different elemental sizes in the potential fracture zone in the middle section of the investigated beam are used in both the ECDM and XFEM for mesh sensitivity investigation. The average elemental side lengths used in the potential fracture zone are $8,4,2$ and $1 \mathrm{~mm}$, respectively. The numerical simulations are carried out without setting the discontinuity evolution path beforehand, so cracks can propagate arbitrarily. Fig. 8 shows predicted deformation after failure by the ECDM using the four different meshes. The approximated failed elements are presented in terms of maximum principal strain (buckled zone is shown in grey), from which a curved feature of the crack can be observed and the predicted crack profiles from four meshes are very similar. At the end of analysis, the exact crack paths predicted by the ECDM with four different meshes together with the experimental envelope are 
plotted as shown in Fig. 9. It can be seen from Fig. 9 that the predicted crack paths from four different meshes are almost identical and certainly within the experimental envelope. In both ECDM and XFEM modelling,crack propagation is determined using a principal stress initiation criterion.

Fig. 10 displays the sequences of predicted failure responses, with progressively refined meshes, provided by the ECDM and XFEM simulations. In these responses, the relative difference of vertical displacements between two sides of the notch, i.e., crack mouth sliding displacement (CMSD), is computed as the abscissa, while the reaction force on the loading point is ordinate. The load - CMSD curves given by the ECDM and XFEM simulations are compared to the experimental solution. It can be seen from Fig. 10 that the ECDM modelling with four different meshes give almost identical responses; the predicted peak load agrees with the experimental envelope very well. In the post-failure softening stage the predicted residual stiffness is slightly lower than the experimental observation because a residual load capacity was still recorded in experimental work due to the compressive stresses around the rigid cap, however, at the later post-failure softening stage, the ECDM prediction has same tendency as with the experimental measurement. This benchmark test modelling indicates that the developed ECDM is capable of predicting arbitrary crack propagation with accuracy and robustness along with the change of mesh size. In contrast, the results of peak load are obviously overestimated by XFEM compared to experimental measurement, and varies to some extent for different meshes. The post-failure softening behavior is also changed to some extent for different meshes compared to experimental observation. This implies that the outcome from XFEM simulation relatively relates to the mesh size in this investigation. Moreover, XFEM simulation tends to overestimate the structural strength at the failure point and at the stage exhibiting the most softening in all four predicted load - CMSD curves. 

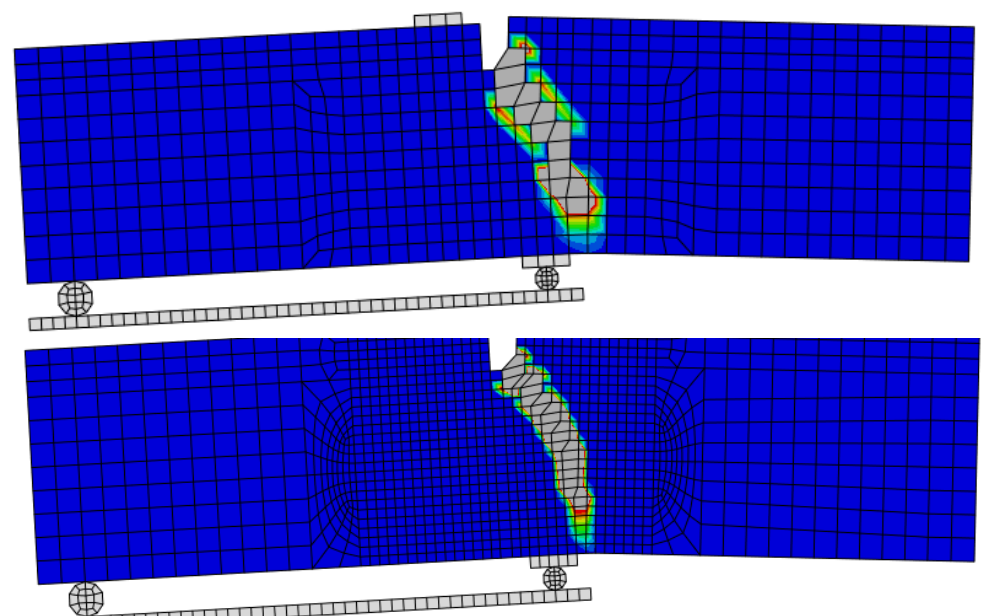

S2

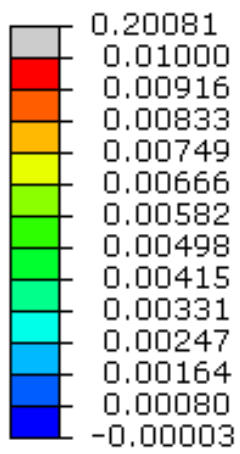

(弗

S3
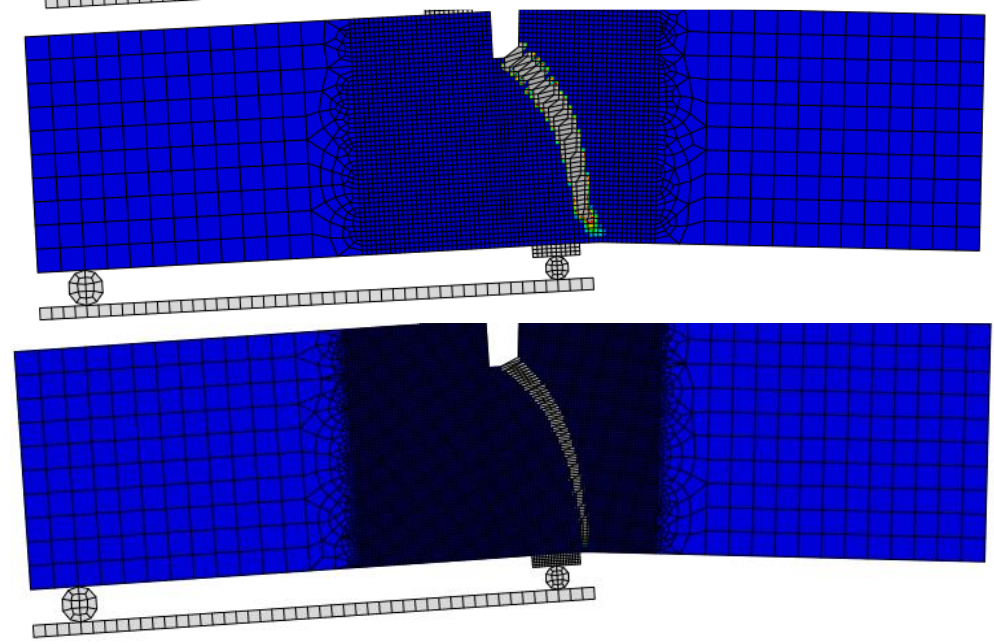

Fig. 8. Predicted crack paths by the ECDM using different meshes.

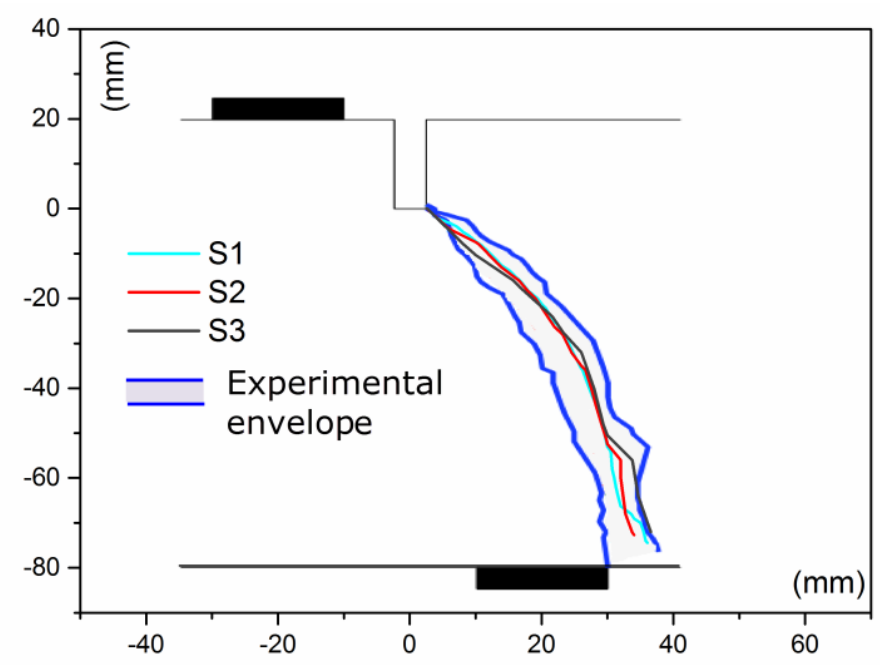

Fig. 9. Predicted crack paths by the ECDM and experimental crack envelope [36]. 

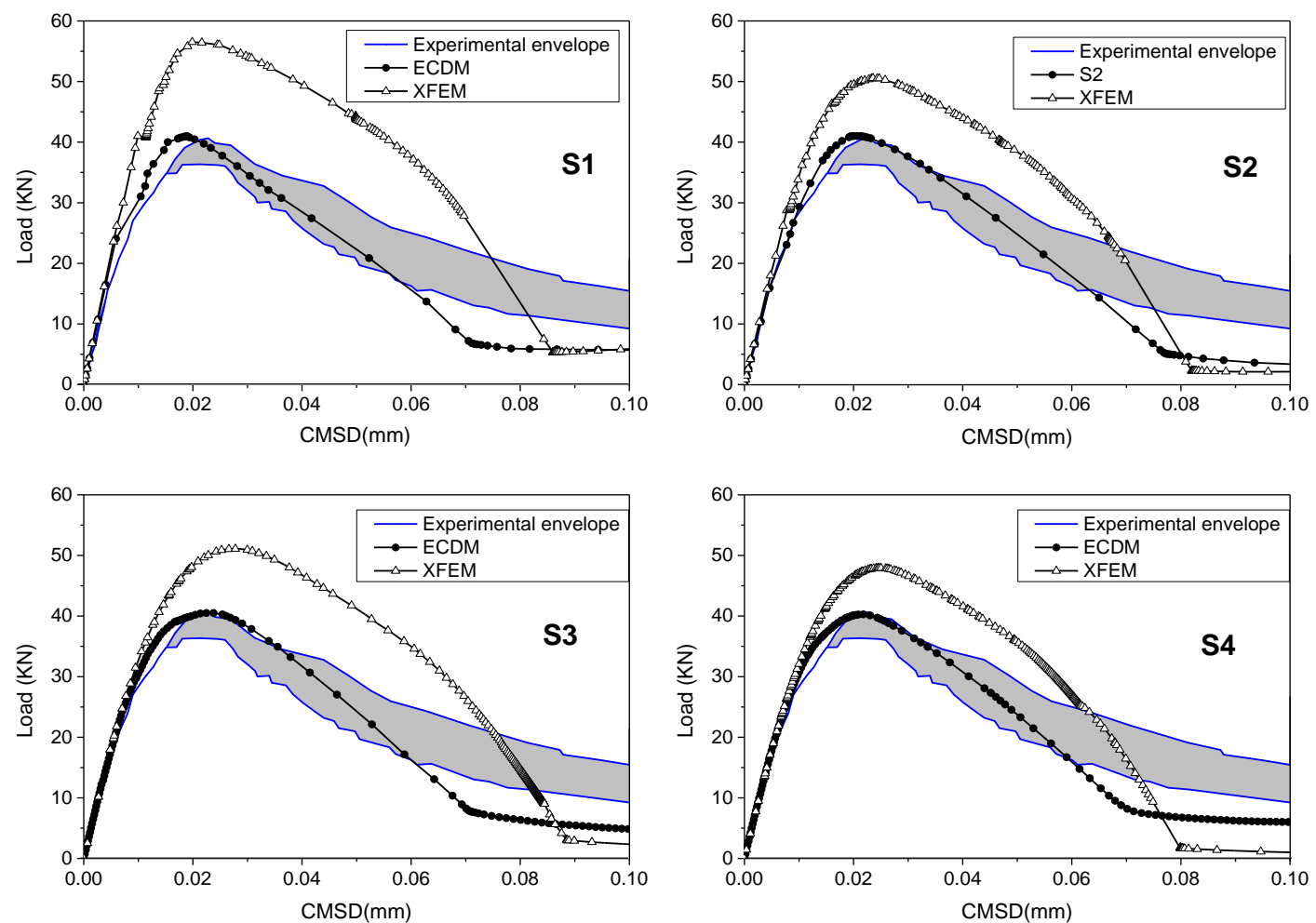

Fig. 10. Load - CMSD curves obtained from the ECDM, XFEM simulations and experimental work [36].

To examine the computational efficiency of the developed ECDM, the total CPU time to solve the nonlinear fracture problem using both ECDM and XFEM in ABAQUS is given in Fig. 11. Plane strain constitutive equations are applied in both the ECDM and XFEM models. Standard gauss integration is performed for each element with 4 integration points. Considering the objectivity of the comparison between the ECDM and XFEM simulations, loading parameters are set to be identical with a maximum prescribed displacement of $0.05 \mathrm{~mm}$, and the initial incremental size and the maximum incremental size (pseudo time increment) are suggested to be 0.01 and 0.1, respectively. Fig. 11a shows the comparison of CPU time between ECDM and XFEM for solving the single notched beam simulation. The CPU time decreases as element size increases. This comparison demonstrates that ECDM can save CPU time by more than $60 \%$ compared to XFEM. It should be noted that the same mesh size is used in both the ECDM and XFEM modelling. In particular, the CPU time from the model S3 is reduced from 1243s to $214 \mathrm{~s}$, which is about $82.7 \%$ CPU time saving. Therefore, ECDM shows significantly improvement in computational efficiency. Fig. $11 \mathrm{~b}$ presents the total iteration number recorded 
for both ECDM and XFEM modelling, from which it can be seen that ECDM offers a great advantage in convergence rate when dealing with nonlinear crack problems compared to XFEM. Particularly, in the model S3 the total iteration number used in ECDM is only $25 \%$ of that spent in XFEM.
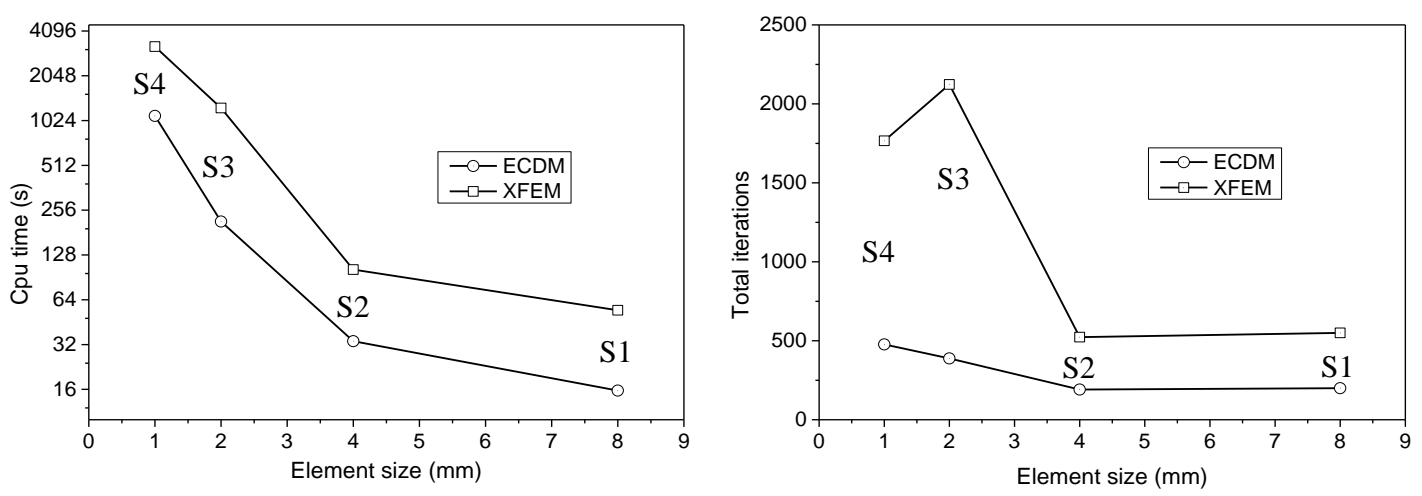

Fig.11. Comparison between ECDM and XFEM simulations for different mesh comparison in (a) CPU time and (b) Total iterations.

\subsection{Double edge notched specimen}

Herein, another benchmark test sample is a double edge notched block, subjected to a mixmode load. The modelling simulation of this test sample is presented for assessing the capability of ECDM in predicting multicrack propagation. Fig. 12 shows a specimen with double notches on the left and right side, tested by Nooru-Mohamed [36], whose configuration and dimensions as well as loading and boundary conditions are presented in Fig. 12. The size of the specimen is $200 \times 200 \times 50 \mathrm{~mm}^{3}$, with two notches $(25 \mathrm{~mm}$ in depth). The steel clamps in dark grey for applying a distributed load are treated as rigid parts in simulation. In experimental work, specimens were tested subjected to different loading conditions. Herein, only the following loading scenario is investigated: a displacement-controlled tensile axial load $F_{n}$ is applied on the top of the specimen; a constant shear force $F_{s}=10 \mathrm{KN}$ is prescribed on the upper left side above the notch of specimen throughout the tensile loading procedure. The following material properties taken from [37] are used in the modelling analysis: Young's modulus $E=3.0 \times$ $104 \mathrm{MPa}$, Poisson's ratio $v=0.2$, tensile strength $f_{t}=3.0 \mathrm{MPa}$ and fracture energy $G_{c}=0.11$ $\mathrm{N} / \mathrm{mm}$. Three meshes with different side lengths of element are used in this investigation, which 
are 2, 4 and $8 \mathrm{~mm}$, respectively. The sequence of these three models are defined as S1, S2 and S3. It should be noted that the existing notches in this specimen are characterized as initial cracks by the cracked ECDM elements.

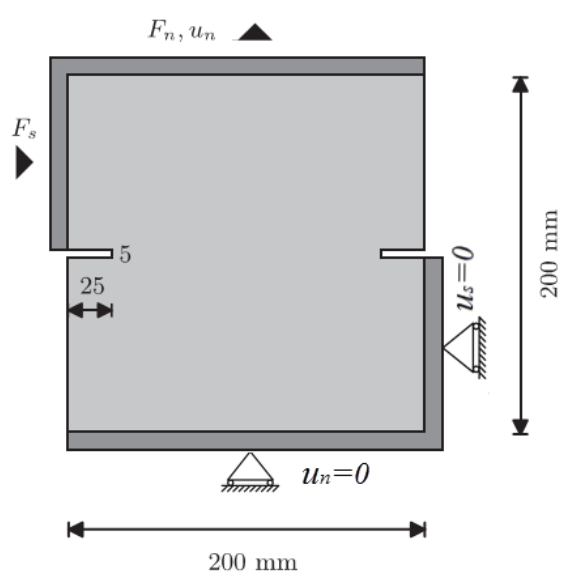

Fig. 12. A mixed-mode fracture test specimen.

Fig. 13(a-c) show the predicted crack propagation by the ECDM simulation in terms of the maximum principal strain contours, from which two highly localized strain zones represent two nearly antisymmetric curved cracks propagating simultaneously from the roots of notches to the opposite sides. It can be seen from Fig. 13(d) that there is an excellent coincidence in crack paths obtained from three models. The comparison between modelling results and experiment measurement can be seen from Fig. 13(d). The ECDM modellings with the three different meshes track the two propagating cracks very well, which fairly coincide with the experimental envelope. There is only a slight difference in the crack paths given by the three different meshes. However, even the coarsest mesh can predict propagation of the two arbitrary cracks. Through the comparison given in Fig. 13, it can be declared that the ECDM approach is able to capture arbitrary multicrack propagation without knowing a priori crack path. This modelling simulation confirms the mesh independence of the developed ECDM approach and the robustness of the crack tracking algorithm. 

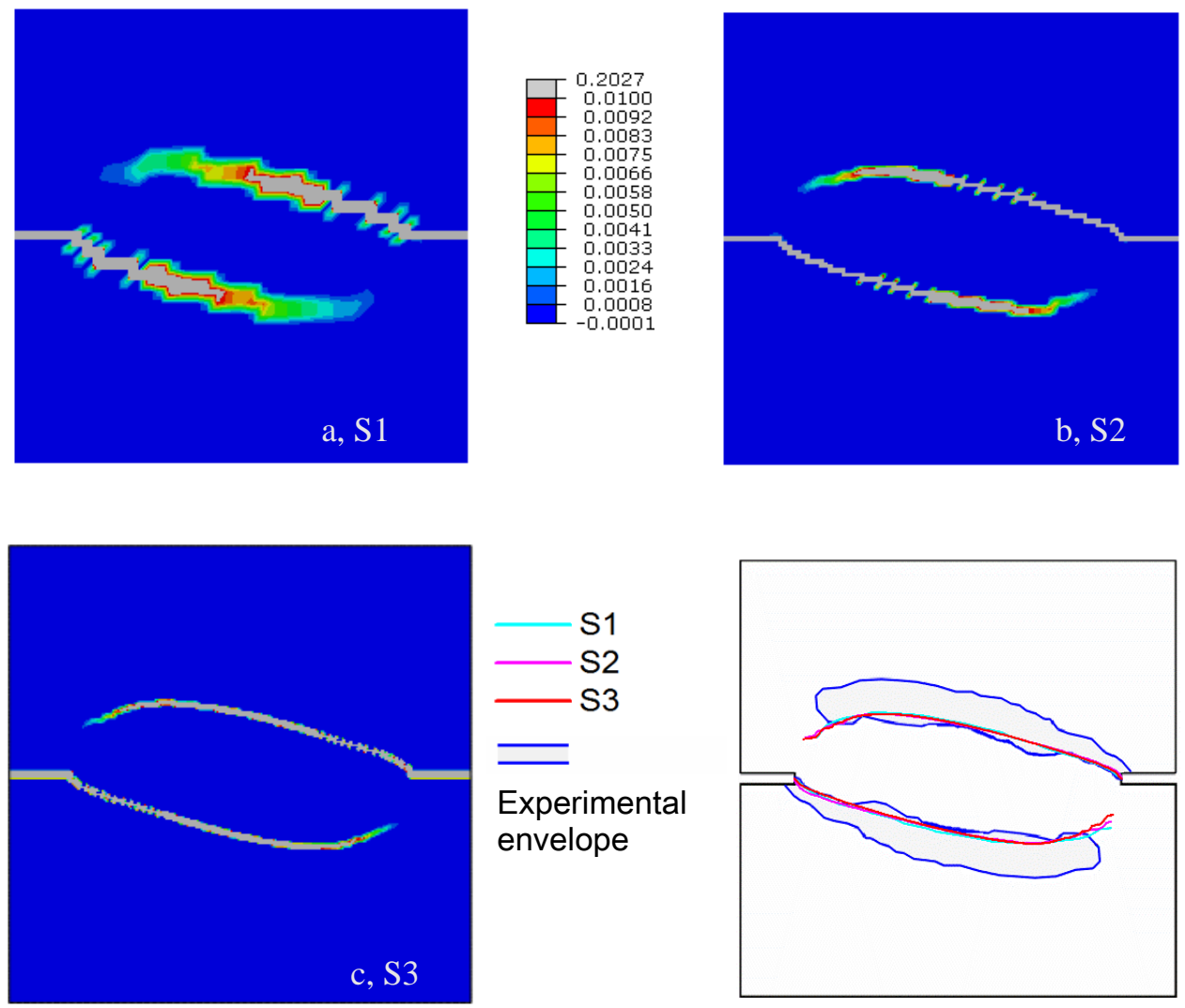

Fig. 13. (a-c): The ECDM predicted multiple crack paths using three different meshes; (d): Comparison between the ECDM and experimental envelope.

Fig.14 shows failure responses recorded with vertical load against the corresponding displacements given by ECDM, XFEM, E-FEM and the experiment work, respectively. The predicted failure responses by ECDM with three meshes are almost coincident, and are very close to simulated responses from XFEM and E-FEM. It can be seen from Fig. 14 that predicted failure points and the post-failure softening behaviour exhibited by the three different modelling approaches are almost identical. A very slight difference can be found during the post-failure softening stage. It should be noted that there is big gap between modelling predictions and experimental work. As discussed in [37] and [38], the experimental measurement obviously overestimated the fracture energy and the tensile strength, which is possibly attributed to the relatively crude characterization of the materials' constitutive relationship. This modeling analysis aims to prove the capability of ECDM in prediction of multicrack propagation with considerably accuracy and robustness compared to XFEM and E-FEM. The computational 
efficiency compared to XFEM is similar to that demonstrated in the last section, and is therefore assumed to held in this and the following sections.

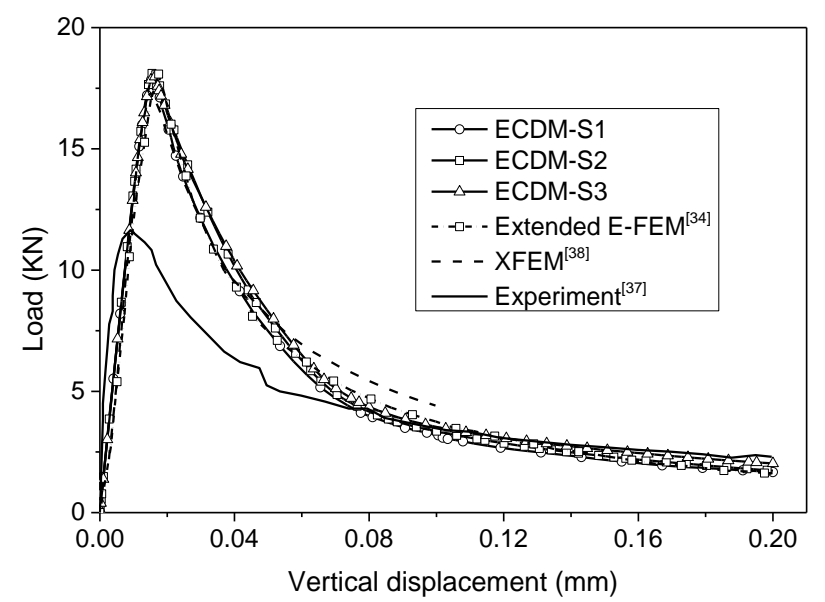

Fig.14. Load - displacement curves given by ECDM, XFEM, E-FEM and experiment.

\subsection{A notched beam under asymmetric bending}

In this section, the capability of the ECDM approach for simulating the crack path of the notched beam subjected to asymmetric bending as reported in [39] is assessed, this being a widely used example for verification of FE numerical algorithms [12, 40]. The geometry and boundary conditions of the investigated beam are illustrated in Fig.15. The following material properties are taken from previous experimental work [40]: The Young's modulus and Poisson's ratio are, respectively, E=38000MPa and v=0.2, material tensile strength $f_{t}=3.0 \mathrm{MPa}$ and fracture energy $G_{c}=70.0 \mathrm{~N} / \mathrm{m}$.

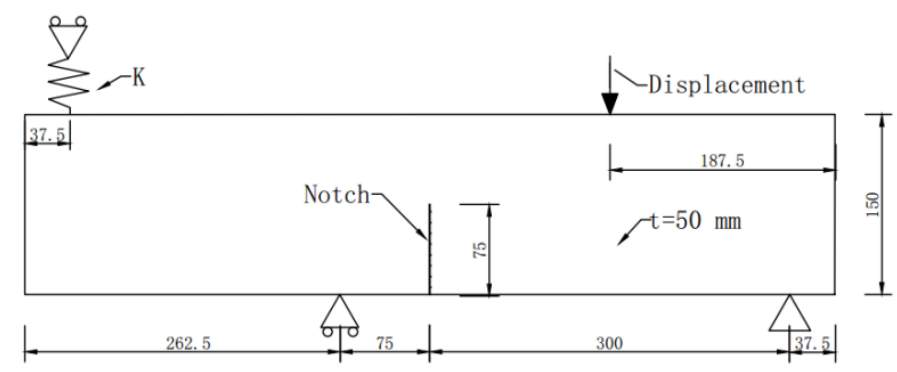

Fig. 15. A beam with a notch under asymmetrical bending.

Fig. 16 shows the ECDM simulated deformation of the investigated beam in the case with a zero coefficient of spring, which means the support at the left top of the beam is 
absent. Crack propagation is presented in Fig. 16 by the maximum principal strain contour, which shows a curved crack path propagated from the top of the initial crack towards the top of the beam. Fig. 17 shows the predicted failure response using the developed ECDM together with the XFEM prediction. A result from experimental work is also given in Fig. 17 for comparison. In Fig.17 (a), the predicted curves of total reaction versus the crack mouth opening displacement are plotted. It can be seen from Fig. 17 that the ECDM predicted initial stiffness agrees well with the results of experimental work. The computed failure load by the ECDM approach is between the upper and lower bound of the failure load envelope obtained from experimental work. In essence, most of the post-failure response given by the two models follows the variation of the experimental envelope. However, the ECDM prediction shows a smoother post-failure response than XFEM. It should be noted that XFEM needs viscous damping in analysis, otherwise no convergent result can be reached. This example demonstrates that the ECDM with embedded micro-mechanical CDM is able to predict the realistic damage propagation in this fracture specimen. Compared with the experimental observation, the ECDM predicted crack path stays within the experimental envelope as shown in Fig. 17b. Fig. 17b also shows XFEM predicted crack path by element $\mathrm{CPE} 4$, which agrees with experimental measurement at early crack path but moves away from experimental measurement at late crack path.

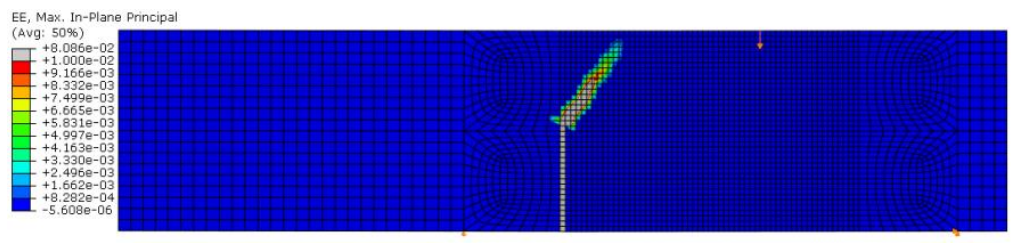

Fig. 16. The ECDM simulated crack propagation in the case of $K=0$.

Fig. 18 shows the ECDM simulation of cracked beam in the case with $\mathrm{K}=\infty$. Comparison of failure responses between the ECDM, XFEM and test in this case is shown in Fig. 19. It can be seen from Fig. 19a that both the ECDM and XFEM predicted initial stiffness agree with the test envelope. The ECDM computed failure load agrees with the tested one, while XFEM 
overestimates the failure load by $15 \%$. In the post failure stage, both the ECDM and XFEM do not follow the tested failure response, and predict higher residual stiffness than the test. The ECDM prediction is between experimental test and XFEM. The reason for this disagreement is possibly due to $\mathrm{K}=\infty$ as an absolute restraint applied on the top left corner of the beam model. The exact same condition would rarely be applied on the test samples. Any slight movement would possibly influence the post failure response. Fig. 19b shows crack paths from the test, and the ECDM and XFEM approaches. The ECDM predicted crack path agrees with the experimental envelope well. The XFEM prediction partly moves away from the tested crack path.
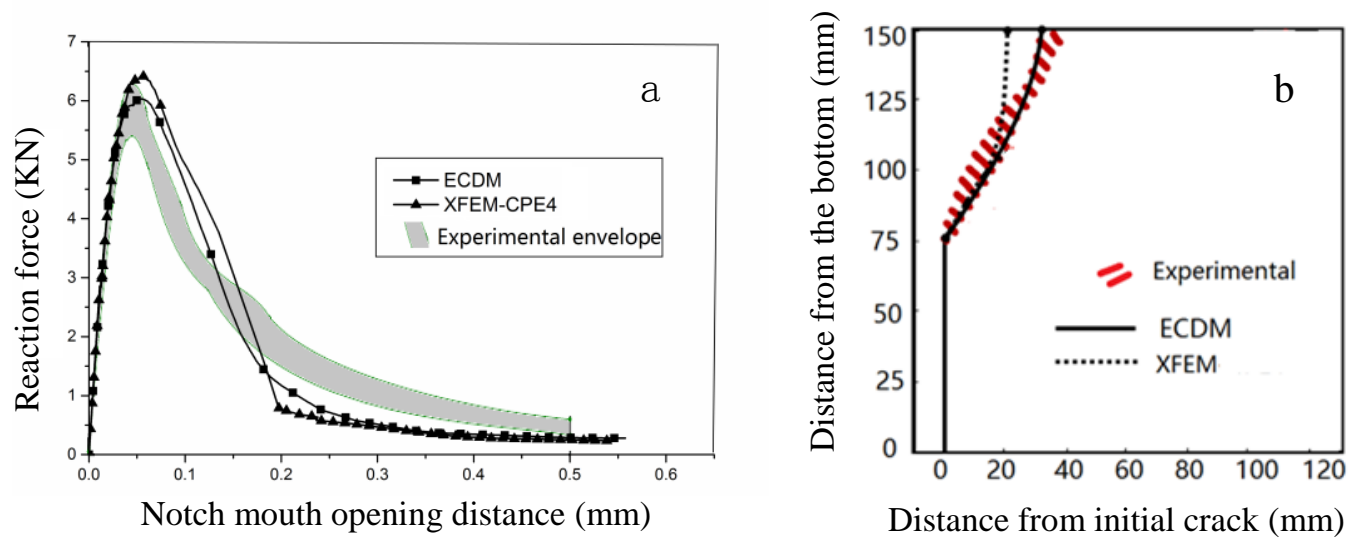

Fig. 17. (a) The ECDM and XFEM simulated failure responses together with experimental envelop, (b) comparison of crack path between predictions and test in the case of $\mathrm{K}=0$.

The above modelling analysis of the beam under asymmetric bending demonstrates that the developed ECDM approach is able to capture the arbitrary crack behaviour with sufficient accuracy, and to characterize the crack path and residual stiffness of the structure during crack propagation. In addition, the convergence and stability of the ECDM approach are comparable to XFEM.

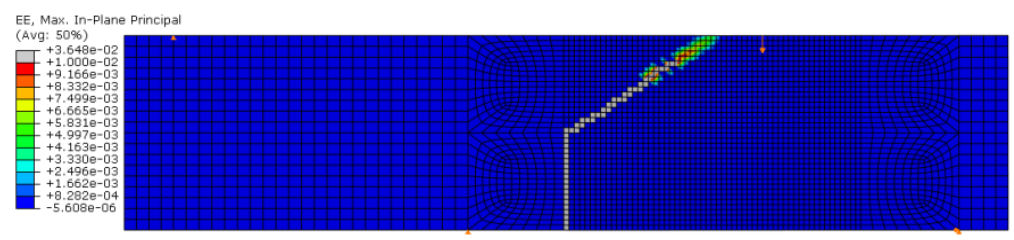

Fig.18. The ECDM simulated crack propagation in the case of $K=\infty$. 

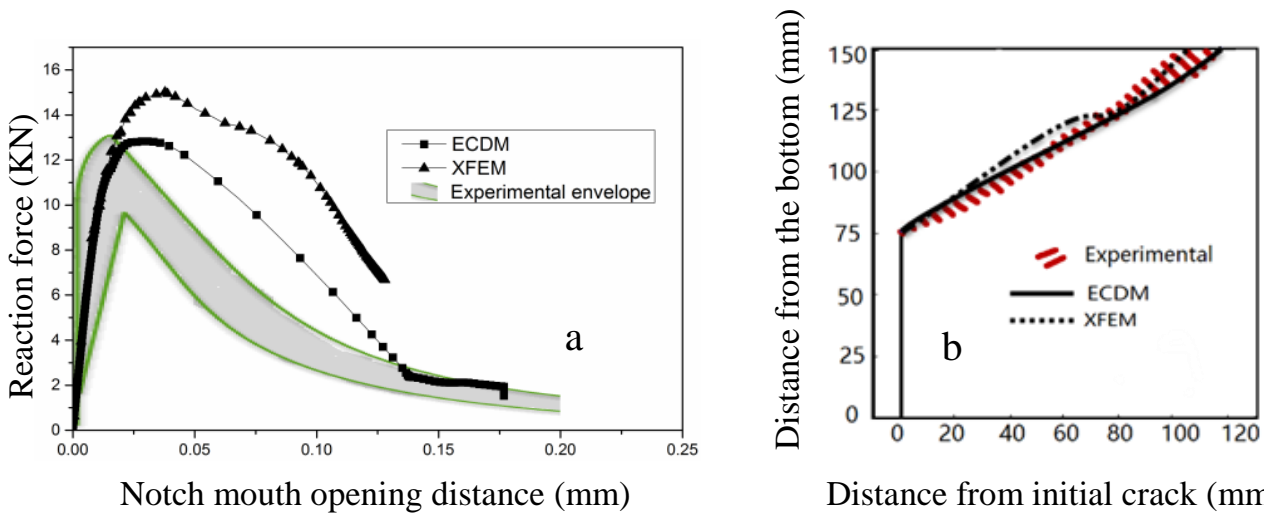

Distance from initial crack (mm)

Fig. 19. (a) Crack mouth opening displacement, (b) comparison of crack path between predictions and test in the case of $K=\infty$.

\section{Conclusion and future work}

This paper presents a study on the performance of the developed ECDM formulation. Starting from using the enriched displacement approximation with shifted Heaviside function, then a condensation is consequently performed for eliminating the enrichments at the elemental level, by which, the developed ECDM approach can describe arbitrary discontinuities without enriched Dofs. A cohesive damage law is used to characterize the failure evolution of quasibrittle materials. Through the introduction of an equivalent damage scalar relating to strain field, the energy dissipation can be consistently maintained during failure evolution. This model, benefiting from hiring standard shape functions, without the need of additional Dofs or nodes, is expected to address the challenge of computational efficiency without sacrificing numerical accuracy. The equivalent damage scalar introduced by this investigation guarantees the constant dissipation of fracture energy which can achieve a more accurate failure load and a post failure response. This developed ECDM has the following specific features: (a) enriched DoFs are eliminated from the fully condensed equilibrium equations; (b) the cohesive damage law is embedded into the condensed equilibrium equation; (c) the effects from the enriched DoFs and the cohesive characteristic are accounted into the final condensed formulation; (d) the derived formulations are presented with the standard FEM displacements only; (e) a novel equivalent damage scalar as a function of strain field is employed based on the thermal dissipation, which enables the model conforms with thermodynamic consistency. The ECDM is a computationally efficient approach since only standard DoFs are needed. 
This paper illustrates the implementation of the ECDM approach as a user element in ABAQUS. Representative numerical benchmark examples, characterized by mixed-mode fracture, are used to validate the performance of the ECDM. Through three fracture benchmark specimens, it has been demonstrated that the developed ECDM achieves a significant improvement in numerical accuracy, efficiency, and robustness. The ECDM approach can achieve highly acceptable solution even with a relatively coarse mesh, while the XFEM in ABAQUS overestimates the structural strength and shows the feature of mesh dependence. In discussion of computational efficiency through investigated examples, the ECDM approach can save great computational cost accounted by CPU time compared to XFEM. This investigation shows the significance of ECDM in computational damage analysis of engineering materials.

This study shows that ECDM is a promising approach with high computational efficiency for predicting multicrack mechanisms in large-scalar structures. Currently, the developed ECDM is within the framework of $2 \mathrm{D}$ plain strain and stress quadrilateral elements. In the future, a ECDM based 3D solid element needs to be implemented. Meanwhile, the future work will include the development of the ECDM based thermal damage model, fatigue damage model and impact damage model. 


\section{Reference}

[1]. Foulk, J.W., D.H. Allen and K.L.E. Helms, Formulation of a three-dimensional cohesive zone model for application to a finite element algorithm. Computer Methods in Applied Mechanics and Engineering, 2000. 183(1-2): p. 51-66.

[2]. Nguyen, V.P., et al., Modelling interfacial cracking with non-matching cohesive interface elements. Computational Mechanics, 2016. 58(5): p. 731-746.

[3]. Reinoso, J. and M. Paggi, A consistent interface element formulation for geometrical and material nonlinearities. Computational Mechanics, 2014. 54(6): p. 1569-1581.

[4]. Távara, L., et al., Cohesive-zone-model formulation and implementation using the symmetric Galerkin boundary element method for homogeneous solids. Computational Mechanics, 2013. 51(4): p. 535-551.

[5]. Murthy, K.S.R.K. and M. Mukhopadhyay, Adaptive finite element analysis of mixed-mode crack problems with automatic mesh generator. International Journal for Numerical Methods in Engineering, 2000. 49(8): p. 1087-1100.

[6]. Park, K., et al., Adaptive mesh refinement and coarsening for cohesive zone modeling of dynamic fracture. International Journal for Numerical Methods in Engineering, 2012. 92(1): p. 1-35.

[7].Zienkiewicz, O.C. and J.Z. Zhu, A simple error estimator and adaptive procedure for practical engineering analysis. International Journal for Numerical Methods in Engineering, 1987. 24(2): p. 337357.

[8]. Fang, X.J., et al., An augmented cohesive zone element for arbitrary crack coalescence and bifurcation in heterogeneous materials. International Journal for Numerical Methods in Engineering, 2011. 88(9): p. 841-861.

[9]. BABUŠKA, I. and J.M. MELENK, THE PARTITION OF UNITY METHOD. International Journal for Numerical Methods in Engineering, 1997. 40(4): p. 727-758.

[10]. Belytschko, T. and T. Black, Elastic crack growth in finite elements with minimal remeshing. International Journal for Numerical Methods in Engineering, 1999. 45(5): p. 601-620.

[11]. Duarte, C.A., et al., A generalized finite element method for the simulation of three-dimensional dynamic crack propagation. Computer Methods in Applied Mechanics and Engineering, 2001. 190(1517): p. 2227-2262.

[12]. Fries, T. and M. Baydoun, Crack propagation with the extended finite element method and a hybrid explicit-implicit crack description. International Journal for Numerical Methods in Engineering, 2012. 89(12): p. 1527-1558.

[13]. Oden, J.T., C.A.M. Duarte and O.C. Zienkiewicz, A new cloud-based hp finite element method. Computer Methods in Applied Mechanics and Engineering, 1998. 153(1-2): p. 117-126.

[14]. Strouboulis, T., I. Babuška and R. Hidajat, The generalized finite element method for Helmholtz equation: Theory, computation, and open problems. Computer Methods in Applied Mechanics and Engineering, 2006. 195(37-40): p. 4711-4731.

[15]. Mo S, N., J. Dolbow and T. Belytschko, A finite element method for crack growth without remeshing. International Journal for Numerical Methods in Engineering, 1999. 46(1): p. 131-150.

[16]. Wells, G.N. and L.J. Sluys, A new method for modelling cohesive cracks using finite elements. International Journal for Numerical Methods in Engineering, 2001. 50(12): p. 2667-2682. 
[17].Zhao, J., Y. Li and W.K. Liu, Predicting band structure of 3D mechanical metamaterials with complex geometry via XFEM. Computational Mechanics, 2015. 55(4): p. 659-672.

[18]. Bhattacharya, S., et al., Fatigue crack growth simulations of interfacial cracks in bi-layered FGMs using XFEM. Computational Mechanics, 2013. 52(4): p. 799-814.

[19]. Giner, E., et al., Extended finite element method for fretting fatigue crack propagation. International Journal of Solids and Structures, 2008. 45(22-23): p. 5675-5687.

[20]. Gerstenberger, A. and W.A. Wall, An eXtended Finite Element Method/Lagrange multiplier based approach for fluid - structure interaction. Computer Methods in Applied Mechanics and Engineering, 2008. 197(19-20): p. 1699-1714.

[21].Zilian, A. and A. Legay, The enriched space - time finite element method (EST) for simultaneous solution of fluid - structure interaction. International Journal for Numerical Methods in Engineering, 2008. 75(3): p. 305-334.

[22]. Ashari, S.E. and S. Mohammadi, Delamination analysis of composites by new orthotropic bimaterial extended finite element method. International Journal for Numerical Methods in Engineering, 2011. 86(13): p. 1507-1543.

[23]. Grogan, D.M., S.B. Leen and C.M. Ó Brádaigh, An XFEM-based methodology for fatigue delamination and permeability of composites. Composite Structures, 2014. 107: p. 205-218.

[24]. Wang, Z., et al., Numerical simulation of crack growth in brittle matrix of particle reinforced composites using the xfem technique. Acta Mechanica Solida Sinica, 2012. 25(1): p. 9-21.

[25]. Grogan, D.M., C.M. Ó Brádaigh and S.B. Leen, A combined XFEM and cohesive zone model for composite laminate microcracking and permeability. Composite Structures, 2015. 120: p. 246-261.

[26]. Areias, P.M.A. and T. Belytschko, A comment on the article "A finite element method for simulation of strong and weak discontinuities in solid mechanics” by A. Hansbo and P. Hansbo [Comput. Methods Appl. Mech. Engrg. 193 (2004) 3523 - 3540]. Computer Methods in Applied Mechanics and Engineering, 2006. 195(9-12): p. 1275-1276.

[27]. Garikipati, K. and T.J.R. Hughes, A variational multiscalar approach to strain localization formulation for multidimensional problems. Computer Methods in Applied Mechanics and Engineering, 2000. 188(1): p. 39-60.

[28]. Rudraraju, S., et al., Predictions of crack propagation using a variational multiscale approach and its application to fracture in laminated fiber reinforced composites. Composite structures, 2012. 94(11): p. 3336.

[29]. Rudraraju, S.S., et al., In-plane fracture of laminated fiber reinforced composites with varying fracture resistance: Experimental observations and numerical crack propagation simulations. International Journal of Solids and Structures, 2010. 47(7-8): p. 901-911.

[30]. Oliver, J., A.E. Huespe and E. Samaniego, A study on finite elements for capturing strong discontinuities. International Journal for Numerical Methods in Engineering, 2003. 56(14): p. 2135 2161.

[31]. Oliver, J., A.E. Huespe and P.J. Sánchez, A comparative study on finite elements for capturing strong discontinuities: E-FEM vs X-FEM. Computer Methods in Applied Mechanics and Engineering, 2006. 195(37-40): p. 4732-4752.

[32]. Wu, J., F. Li and S. Xu, Extended embedded finite elements with continuous displacement jumps for the modeling of localized failure in solids. Computer Methods in Applied Mechanics and Engineering, 
2015. 285: p. 346-378.

[33]. Gigliotti, L., Assessment of the applicability of XFEM in Abaqus for modeling crack growth in rubber. Applied Mechanics, 2012. Master thesis.

[34]. Li, X. and J. Chen, An extended cohesive damage model for simulating arbitrary damage propagation in engineering materials. Computer Methods in Applied Mechanics and Engineering, 2017. 315: p. 744-759.

[35]. Chen, J., An extended cohesive damage model with a length scalar in fracture analysis of adhesively bonded joints. Engineering Fracture Mechanics, 2014. 119: p. 202-213.

[36]. Nooru-Mohamed, M.B., Mixed-mode fracture of concrete: an experimental approach. 1992, Delft University of Technology: Delft.

[37]. Dumstorff, P. and G. Meschke, Crack propagation criteria in the framework of X-FEM-based structural analyses. International Journal for Numerical and Analytical Methods in Geomechanics, 2007. 31(2): p. 239-259.

[38]. Feist, C. and G. Hofstetter, An embedded strong discontinuity model for cracking of plain concrete. Computer Methods in Applied Mechanics and Engineering, 2006. 195(52): p. 7115-7138.

[39]. Gálvez, J.C., et al., Fracture of concrete under mixed loading-experimental results and numerical prediction. Proceedings of FRAMCOS, 1998. 3(2): p. 729-38.

[40]. Gürses, E. and C. Miehe, A computational framework of three-dimensional configurational-forcedriven brittle crack propagation. Computer Methods in Applied Mechanics and Engineering, 2009. 198(15): p. 1413 - 1428. 